San Jose State University

SJSU ScholarWorks

Master's Theses

Master's Theses and Graduate Research

Fall 2015

\title{
Quantifying Variability in Oculomotor and Manual Choice Response Times
}

Angelica Godinez

San Jose State University

Follow this and additional works at: https://scholarworks.sjsu.edu/etd_theses

\section{Recommended Citation}

Godinez, Angelica, "Quantifying Variability in Oculomotor and Manual Choice Response Times" (2015).

Master's Theses. 4636.

DOI: https://doi.org/10.31979/etd.jefb-547c

https://scholarworks.sjsu.edu/etd_theses/4636

This Thesis is brought to you for free and open access by the Master's Theses and Graduate Research at SJSU ScholarWorks. It has been accepted for inclusion in Master's Theses by an authorized administrator of SJSU ScholarWorks. For more information, please contact scholarworks@sjsu.edu. 


\title{
QUANTIFYING VARIABILITY IN OCULOMOTOR AND MANUAL CHOICE RESPONSE TIMES
}

\author{
A Thesis \\ Presented to \\ The Faculty of the Department of Human Factors and Ergonomics
}

San José State University

In Partial Fulfillment

of the Requirements for the Degree

Master of Science

by

Angelica Godinez

December 2015 
(C) 2015

Angelica Godinez

ALL RIGHTS RESERVED 
The Designated Thesis Committee Approves the Thesis Titled

QUANTIFYING VARIBILITY IN OCULOMOTOR AND MANUAL CHOICE RESPONSE TIMES

by

Angelica Godinez

\section{APPROVED FOR THE DEPARTMENT OF HUMAN FACTORS AND ERGONOMICS}

SAN JOSÉ STATE UNIVERSITY

December 2015

Dr. Kevin Jordan

Department of Psychology

Dr. Dorion Liston

Department of Psychology

Dr. Emily Wughalter

Department of Kinesiology 


\section{ABSTRACT \\ QUANTIFYING VARIABILITY IN OCULOMOTOR AND MANUAL CHOICE RESPONSE TIMES}

By Angelica Godinez

Response times (RTs) of various motor systems have traditionally been used to characterize aspects of human performance (e.g., fatigue, disease states). However, the properties and sensitivity of different motor systems to detect changes in neural states across multiple timescales remain an open question. In this thesis, we attempt to characterize the difference in sensitivity of the pursuit, saccadic, and manual systems to detect changes in stimulus strength. In Experiment 1, we used a modified Yes-No task to test the effects of contrast $(5,10,20,40,80,100 \%)$ on three pursuit, saccadic, and manual RT's for three observers. In Experiment 2, we used a 2-AFC task to test the effects of luminance (0-10 d' above background noise) on saccadic and manual RT for five observers. We observed: 1) saccadic RT are better correlated with changes in stimulus strength, 2) manual responses are more variable, 3) trial-by-trial variability is greater than variability across sessions, and 4) each pair of motor systems shows significant shared variability. We conclude that oculomotor and manual responses have different signal processing and RT characteristics, and may have different levels of utility to detect physiological factors that affect performance (e.g., Dinges \& Powell, 1985), with the saccadic system being more sensitive to changes in stimulus strength and less variable in the timing of the response. 


\section{ACKNOWLEDGEMENTS}

I would like to thank my mentor and PI, Dr. Liston, for his invaluable guidance and support. I could not have asked for a better mentor. Also, Rami Ersheid for his technical support and Dr. Stone for his help and support throughout the project. In addition, I would like to thank Dr. Jordan for his counsel in writing the thesis and Dr. Wughalter for her time and expertise. Lastly, I would like to thank Lily Wong for her help with editing the document and my family for supporting me without really understanding what I do. This project was supported by NSF's Program in Perception, Action and Cognition Program (NSF 0924841) and the National Space Biomedical Research Institute (SA 2002). 


\section{TABLE OF CONTENTS}

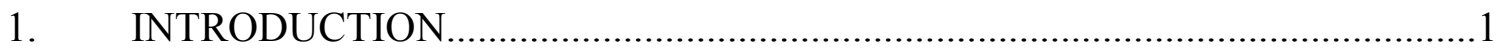

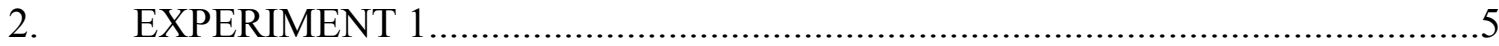

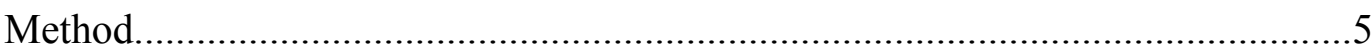

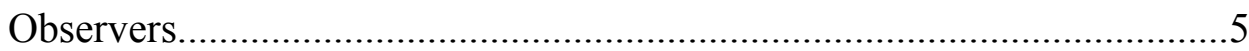

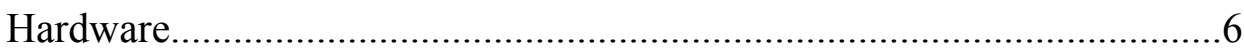

Stimulus...................................................................................

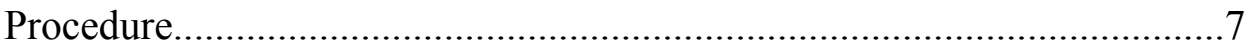

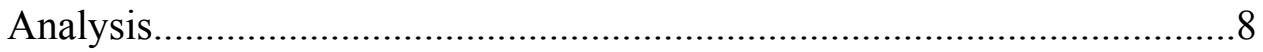

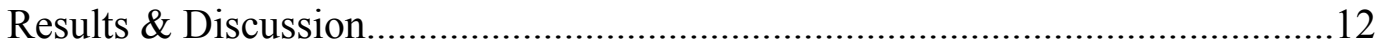

Effects of contrast on RT ..................................................................13

Shared variability across motor systems........................................ 14

Variability within and across-sessions..............................................14

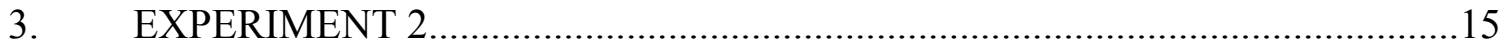

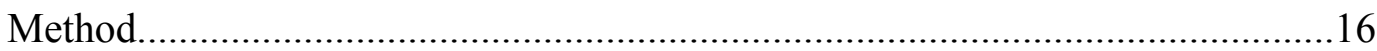

Observers............................................................................... 16

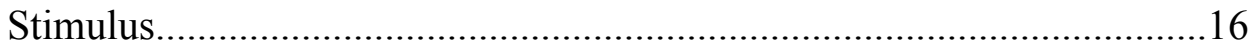


Procedure

Analysis

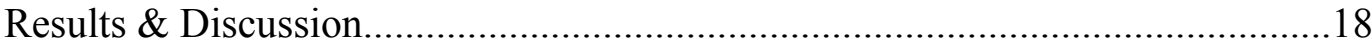

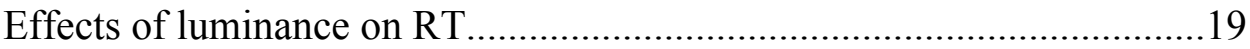

Shared variability across motor systems.........................................20

Variability within and across-sessions...........................................21

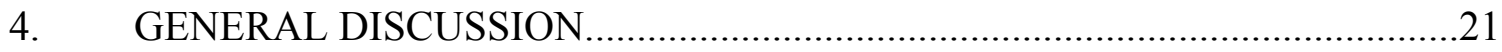

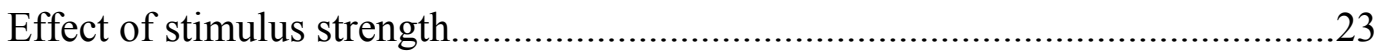

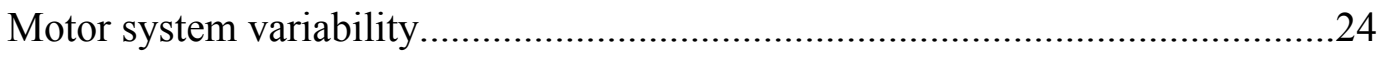

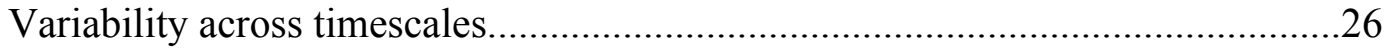

Shared neural noise.................................................................................2

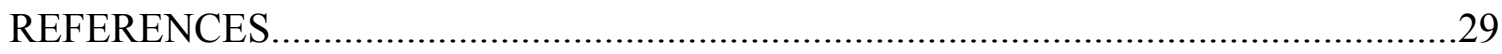

Appendix A. NASA Ames Minimal Risk Consent Form............................35

Appendix B. NASA Ames Research Center IRB Approval Experiment 1..................36

Appendix C. San José State University IRB Approval Experiment 1....................37

Appendix D. NASA Ames Research Center IRB Approval Experiment 2.............38

Appendix E. San José State University IRB Approval Experiment 2................39 


\section{LIST OF TABLES}

1. Across and within-session variability in motor RT for Experiment $1 \ldots \ldots \ldots . . .12$

2. Across and within-session variability in motor RT for Experiment 2........19 


\section{LIST OF FIGURES}

1. Experiment 1 schematic diagram of one trial..........................

2. Effect of stimulus contrast on pursuit, saccadic, and manual latency..........9

3. Correlation between stimulus strength and motor response time.............10

4. Within-session and across-session shared motor system variability..........11

5. Shared across-session variability between stimulus strength and RT ........20 


\section{CHAPTER 1}

\section{INTRODUCTION}

The purpose of this thesis is to quantify response time (RT) variability of various motor systems (pursuit, saccadic, and manual) to changes in stimulus contrast (Experiment 1) and luminance (Experiment 2), variability across multiple time scales, and shared variability between each motor system. RT refers to the time between the presentation of a stimulus and the initiation of a response. Quantifying the shared and unshared variability of oculomotor and manual responses can provide insight into the sensitivity of each motor system to varying changes in stimulus strength and ultimately the utility of each system to detect physiological factors that impact human performance.

Motor behavior provides a straightforward means to measure various sensory and motor factors that impact human performance. Indeed, motor RTs show changes related to: sleep deprivation (Basner, Mollicone, \& Dinges, 2011; Dinges \& Powell, 1985; Heaton, Maule, Maruta, Kryskow, \& Ghajar, 2014; Lisper \& Kjellberg, 1972), hypoxia (Fowler \& Prlic, 1995; Kobrick \& Dusek, 1970; Kobrick, 1972), fatigue (Ting, Hwang, Doong, \& Jeng, 2008; Welford, 1980), vigilance (Buck, 1966), pharmacological toxicity (Liguori, Gatto, \& Robinson, 1998; Tzambazis \& Stough, 2000), sympathetic nervous system arousal (Rogers, Phan, Kennaway, \& Dawson, 1998; Wilkinson \& Houghton, 1982), autoimmune disease (Martin et al., 1992), hypotension (Duschek, Weisz, \& Schandry, 2003), neurodegenerative disease (Anderson \& MacAskill, 2013; Garbutt et al., 2008; Van Stockum, Macaskill, Myall, \& Anderson, 2013), head impact trauma 
(Heitger et al., 2004; Hicks \& Birren, 1970; Pearson, Armitage, Horner, \& Carpenter, 2007), and space-motion sickness (Ratino, Repperger, Goodyear, Potor, \& Rodriguez, 1988). In some cases, motor RT increases linearly with severity of the condition or internal state (e.g., sleep deprivation, fatigue, hypotension, neurodegenerative disease, head-impact trauma, and space-motion sickness), while in other cases, motor RT decreases with severity of the condition or internal state (e.g., stimulants and arousal). However, in terms of performance, RT is a factor of the Yerkes-Dodson law, in which optimum performance is a function of arousal, where too little or not enough arousal leads to poor performance (e.g., vigilance and sympathetic nervous system arousal). Traditionally, motor RTs have been utilized as a metric for detection of such conditions or states.

The motor systems queried by these tests often vary, and include manual button press (Basner et al., 2011; Dinges \& Powell, 1985; Wilkinson \& Houghton, 1982), saccadic (Pearson et al., 2007), and smooth pursuit (Liston \& Stone, 2014) responses. In addition to absolute RTs, many other properties of motor behavior may be useful indicators of changes in neural states, including: RT variability (Kobrick \& Dusek, 1970; Kobrick, 1972; Lisper \& Kjellberg, 1972), changes in saccadic peak velocity (Hikosaka, Nakamura, \& Nakahara, 2006; Miller, 2004; Uri, Linder, Moore, Pool, \& Thornton, 1989), fixation duration (Abadi \& Gowen, 2004), antisaccades (Garbutt et al., 2008), baseline pupil size (Miller, 2004), and saccade gain (Garbutt et al., 2008). Across motor systems (e.g., saccadic, smooth pursuit, manual), the properties (e.g., timing) and sensitivity (e.g., accurate, low signal-to-noise ratio) of behavior to factors that impact 
human performance and ultimately provide insight into neural states remain an open research area; if one is to use motor RT as a measurement of internal changes, which system is best suited for the job?

The earliest RT studies show a strong effect of stimulus strength (Cattell, 1886; Henmon, 1906; Jastrow, 1890) on motor RTs, shortening the time it takes to respond as stimulus strength increases. However, the earliest RT studies measured either verbal (Jastrow, 1890) or manual responses (Buck, 1966; Cattell, 1886). Oculomotor responses, which have been tested more recently due to advances in eye-tracking techniques (Yarbus, 1967) show effects of stimulus contrast (Beutter, Eckstein, \& Stone, 2003; Carpenter, 2004; Liston \& Krauzlis, 2003; Masson, Mestre, Blin, \& Pailhous, 1994; Taylor, Carpenter, \& Anderson, 2006; Wheeless, Cohen, \& Boynton, 1967), luminance (Bompas \& Sumner, 2008; Kane, Wade, \& Ma-Wyatt, 2011; Liston, Krukowski, \& Stone, 2013; Masson et al., 1994; Wheeless et al., 1967), target speed (Rashbass, 1961; Robinson, 1964, 1965), and changes in target position (Kane et al., 2011). Similarly, manual RTs show effects of contrast (Taylor et al., 2006), luminance (Bompas \& Sumner, 2008; Cattell, 1886; Kane et al., 2011), and changes in target position (Kane et al., 2011). However, paired comparisons of various motor systems show manual RTs to visual stimuli have longer latencies (Beutter et al., 2003; Bompas \& Sumner, 2008; Kane et al., 2011; Stone \& Krauzlis, 2003) and take longer to modify compared to oculomotor responses (Kane et al., 2011). Could this delay in RT potentially be advantageous in detecting internal changes or simply a greater source of variability? 
Paired RT comparisons of various motor systems also show systematic differences in their properties (e.g., Beutter et al., 2003; Bompas \& Sumner, 2008; Kane et al., 2011; Liston \& Krauzlis, 2003; Masson et al., 1994), which suggests that although there are shared neural processing stages (Beutter \& Stone, 2000; Krauzlis \& Adler, 2001; Krauzlis \& Stone, 1999; Stone \& Krauzlis, 2003), manual and oculomotor responses may ultimately display different utility to detect internal changes. Indeed, when stimulus strength is high, oculomotor and manual responses are correlated on a trial-by-trial basis (Beutter \& Stone, 2000; Krauzlis \& Adler, 2001). However, when stimulus strength is low, oculomotor and manual responses no longer share the same trial-by-trial correlation (Beutter \& Stone, 2000), which suggests that one of the two systems may be more sensitive to small internal changes. Thus, the current literature contains an emerging picture of the differences in signal processing mechanisms (Beutter et al., 2003; Masson et al., 1994; Stone \& Krauzlis, 2003), sensitivity to factors that fluctuate across sessions (Rogers et al., 1998; Sahin, Wood, Plitnick, \& Figueiro, 2014), and underlying physiological circuits that contribute to RT variability (Flykt, 2005; Jennings \& Wood, 1977).

The goal of the present paper is to quantify across and within-session RT variability, shared variability across motor systems, and the responsiveness of various motor systems to detect changes in stimulus contrast and luminance. We used a large range in stimulus contrast (5-100\%) for Experiment 1 and a small range in stimulus luminance (0-10 d' above the background noise) for Experiment 2. Documenting the RT of various motor systems and the variability shared across systems can provide insight 
into the sensitivity of each motor system to varying changes in stimulus strength and the utility of each system to detect internal factors that impact performance.

\section{CHAPTER 2}

\section{EXPERIMENT 1}

The first experiment tested the shared RT variance of the responses of manual and two oculomotor systems (pursuit and saccadic) to changes in stimulus contrast $(5,10,20$, $40,80,100 \%$ ) on a choice RT task. Our method required observers to make a saccade to the stimulus, follow the stimulus in the appropriate direction (pursuit), and indicate the direction of travel by either pressing the left or right button on the mouse. Observers were instructed to respond as quickly and accurately as possible.

\section{Method}

Observers. Three adult observers provided informed consent (Appendix A) to participate in the experiment (all three female, age range: 22-23 years). Observers were required to have normal or corrected-to-normal 20/20 visual acuity. Each observer completed a total of 23 sessions, the first three of which were training sessions. The data used in the analysis were collected in the last 20 sessions, each of which consisted of 400 trials. The protocol used was approved by NASA Ames Research Center Human Institutional Review Board (IRB) (Appendix B) and San José State University Human IRB (Appendix C). Our methods adhered to the Helsinki Declaration. 
Hardware. Experiments 1 and 2 were executed using a Linux computer running custom-built software that controlled the timing of the experiment, displayed the stimuli, acquired eye-position data, and saved it to a disk. All display events were recorded in an output data file, allowing precise $(1 \mathrm{~ms})$ registration with the eye-position data. Stimuli were displayed on an EIZO FlexScan T966 monitor with $1024 \times 768$ resolution $(40 \mathrm{~cm}$ horizontal by $29.5 \mathrm{~cm}$ vertical), $60 \mathrm{~Hz}$ flicker rate, calibrated to have a maximum luminance of $80.0 \mathrm{~cd} / \mathrm{m}^{2}$, and gamma-corrected using a lookup table. The monitor was $47 \mathrm{~cm}$ from the observers, with a viewing angle of $46^{\circ}$ horizontal by $34.8^{\circ}$ vertical.

We sampled eye position at $240 \mathrm{~Hz}$ with a custom-built ISCAN eye tracker. The eye-position traces were calibrated with six parameters (gain, offset, and cross-terms for horizontal and vertical position) (Beutter \& Stone, 1998) fit to the raw digital values for fixations at nine screen locations, which yielded a sample precision of better than $0.3^{\circ}$ (SD of eye position during fixation of calibration points). In the offline analysis, saccades were detected by taking the correlation between a saccade-shaped velocity template and the horizontal eye velocity trace (Liston et al., 2013).

Stimulus. The independent variable in the stimulus was the level of contrast (5, $10,20,40,80,100 \%)$. The stimulus consisted of elongated contrast strips, which were offset vertically and moved horizontally to the left if the stimulus was presented below the fixation point or to the right if the stimulus was presented above the fixation point (Liston \& Krauzlis, 2003, 2005). To avoid prediction of stimulus onset (Luce, 1986; Palmer, Huk, \& Shadlen, 2005), fixation duration was drawn from a truncated exponential distribution (200-5000 ms, $M=700 \mathrm{~ms}$ ). As a psychophysical task, we used 
a slightly modified YES-NO task (Green \& Swets, 1966), where each trial contained a single stimulus drawn from two orthogonal parametric categories: horizontal motion either "leftward" or "rightward" and location either "above" or "below" fixation. As a RT task, this is classified as a choice RT (Luce, 1986; Welford, 1980) paradigm because our stimulus required the observer to initiate one of two responses, either leftward or rightward smooth pursuit, an upward or downward saccadic movement, and a manual button press of either the left or right mouse button for each trial.

Procedure. Observers sat in front of the EIZO FlexScan T966 monitor on a bite bar to keep the head relatively stable. On each trial, observers were instructed to fixate a central cross for a randomized (200-5000 ms) duration (Luce, 1986; Palmer et al., 2005). After fixation, the stimulus appeared either above the fixation point and moved rightward or below the fixation point and moved leftward across the screen (see Figure 1).

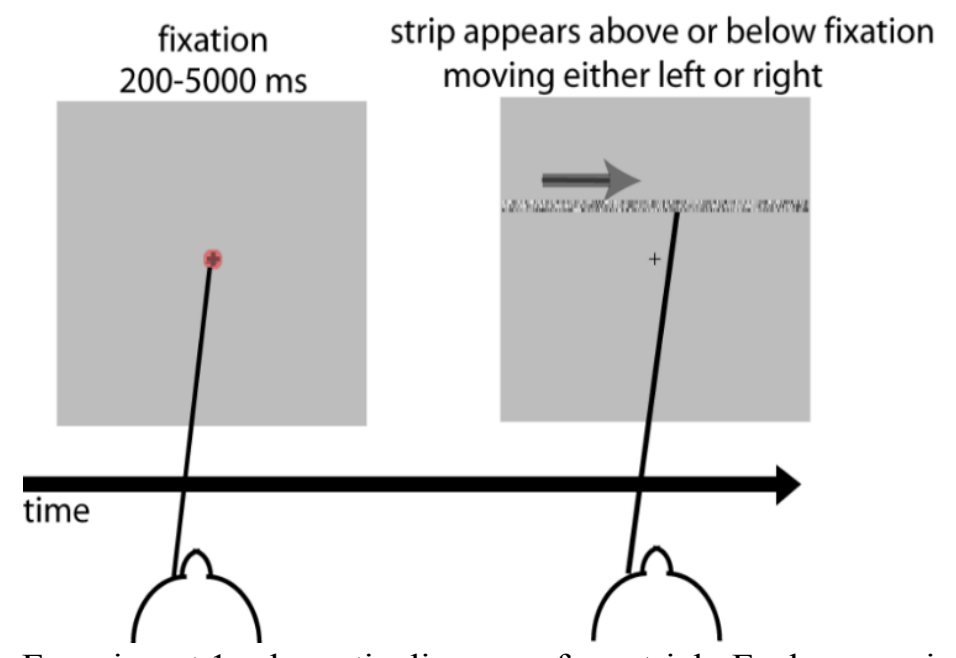

Figure 1. Experiment 1 schematic diagram of one trial. Each screen image represents the central portion $\left(11.7^{\circ}\right.$ horizontal $\times 8^{\circ}$ vertical $)$ of the video monitor. The observer begins by fixating (transparent red circle) a central fixation cross for a randomized duration. A long horizontal strip then appears either above or below the fixation location, moving either rightward (solid gray arrow) or leftward (Liston \& Krauzlis, 2003, 2005). The observer then makes a vertical saccade and horizontal smooth pursuit (transparent red arrow) to stabilize the image of the moving strip on the fovea. 
Observers were instructed to look at and follow the stimulus until it was no longer visible, which required both a pursuit and saccadic response. Observers were also instructed to make a manual button-press response using a generic computer mouse to indicate the direction of the chosen stimulus. Lastly, observers were instructed to respond to the stimulus as fast and accurately as possible.

Analysis. To quantify the effect of contrast on motor system latency we performed a 2-way ANOVA on RT across contrast conditions (5, 10, 20, 40, 80, 100\%) for each motor system (pursuit, saccade, and manual). We then fit the contrast RT data using a linear fit for each motor system and observer (see Figure 2), similar to other loglinear fits of contrast (Bartlett \& Macleod, 1954; Carpenter, 2004; Hyman, 1953) or probability (Hick, 1952). Each fitted line consists of an offset $\left(\mathrm{T}_{0}\right)$ and a contrastdependent slope $(\mathrm{k})$. Since the offset and rate of change were idiosyncratic and motor system dependent, each observer was fitted with a different $\mathrm{T}_{0}$ and $\mathrm{k}$ per motor system.

$$
R T=T_{0}-k \log _{10} \text { (contrast) }
$$



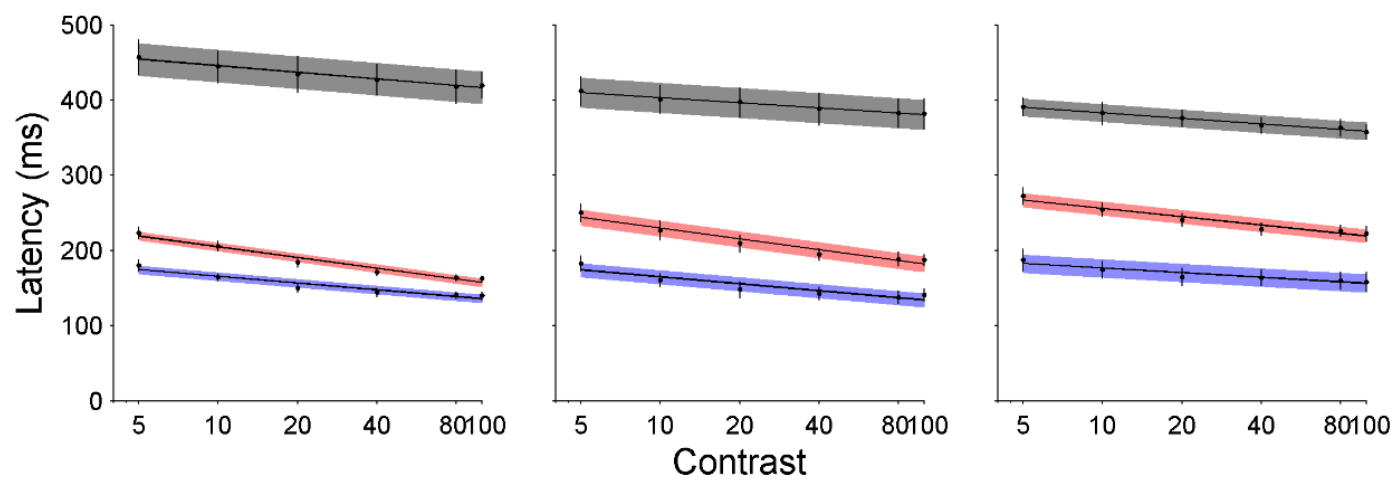

Figure 2. Effect of stimulus contrast on pursuit, saccadic, and manual latency. Each black filled circle plots mean response time across twenty sessions as a function of stimulus contrast, for one motor system, for one observer; error bars represent the standard deviation across twenty sessions. Solid black lines plot the linear regression of latency as a function of log contrast (Carpenter, 2004). Filled colored regions plot the across-session variability, averaged across contrast conditions, for pursuit (blue), saccadic (red), and manual (gray) motor responses. Each set of axes plot data from one observer.

Since our RT data were nonparametric, we used the median as a measure of central tendency. This yielded a set of 20 measurements (one for each session) of median RT for each motor system and observer. These measurements contained variability due to across-session factors that were not explicitly controlled (e.g., circadian rhythm, sleep quality, blood sugar, autonomic arousal, fatigue, etc.). To quantify across-session RT variability, we performed a 2-way ANOVA on RT for motor system and contrast level across all 20 sessions for each observer. We then correlated reciprocal latency with log contrast level across all sessions for each motor system (Figure 3A-C). To quantify shared variability across motor systems and sessions, we calculated the Pearson's r values for each pair of motor system and stimulus strength (Figure 3D-E). 

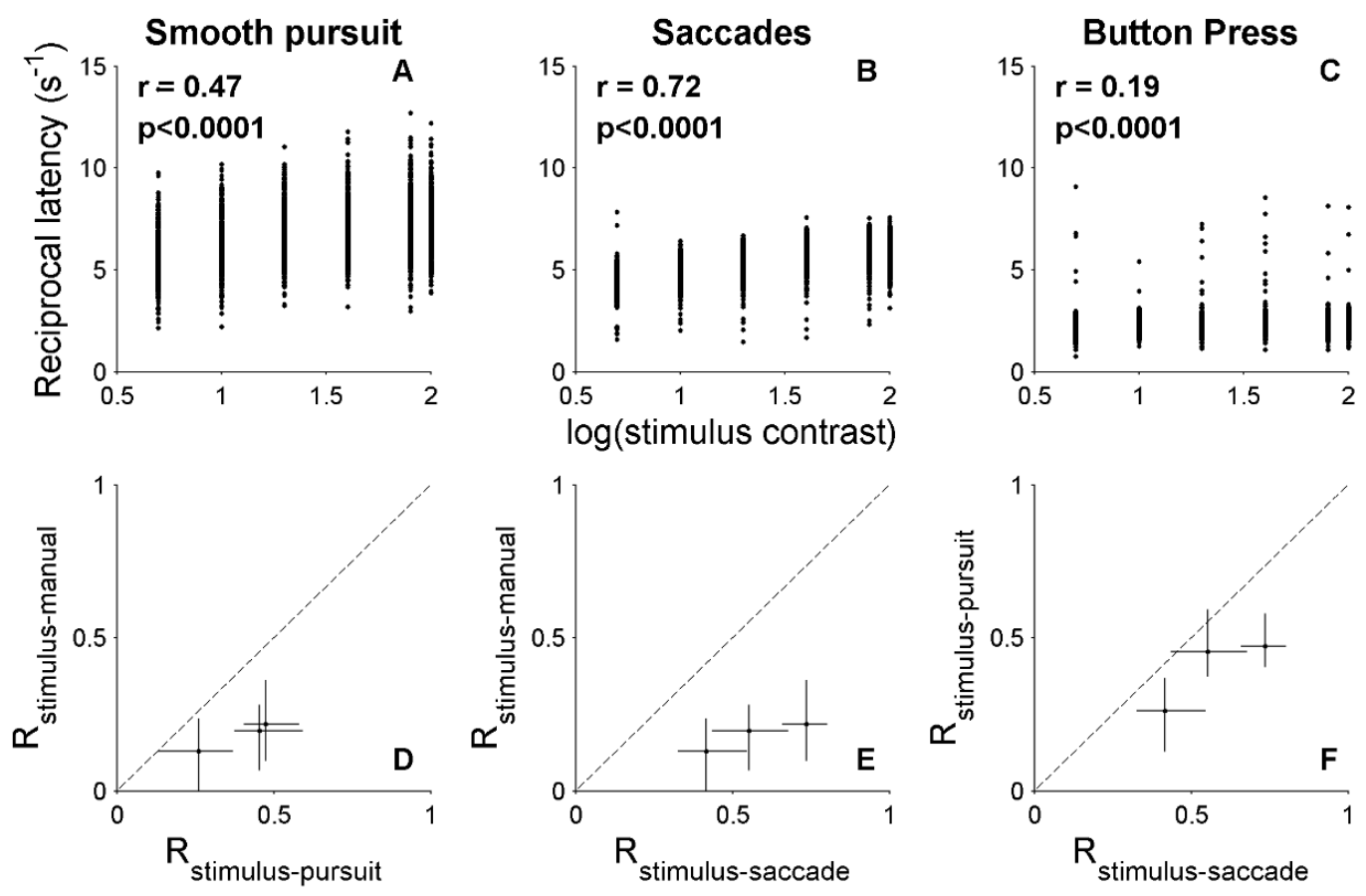

Figure 3. Correlation between stimulus strength and motor response time. Filled circles in A-C plot individual measurements of reciprocal latency as a function of $\log$ stimulus strength for smooth pursuit (A), saccades (B), and button-press (C) responses for one observer. Data for all twenty sessions for one observer are shown, and the Pearson's $r$ value across all measurements is inset. Each filled circle in D-F plots the Pearson's $r$ value for two motor systems for one observer, averaged across all twenty sessions. Error bars represent the central $95 \%$ of the distribution of across-session measurements.

To quantify within-session RT variability, we fit RT measurements as a function of contrast with a linear regression, for each motor system and observer. We then subtracted the expected RT for each contrast level from the measured values, to yield a set of residual RT measurements, again due to within-session factors that were not explicitly controlled (e.g., heart rate, blood sugar, attention modulations, boredom, fatigue, motivation). We then correlated the set of residual measurements for each pair of motor systems, for each of the 20 sessions across observers (see Figure 4). 


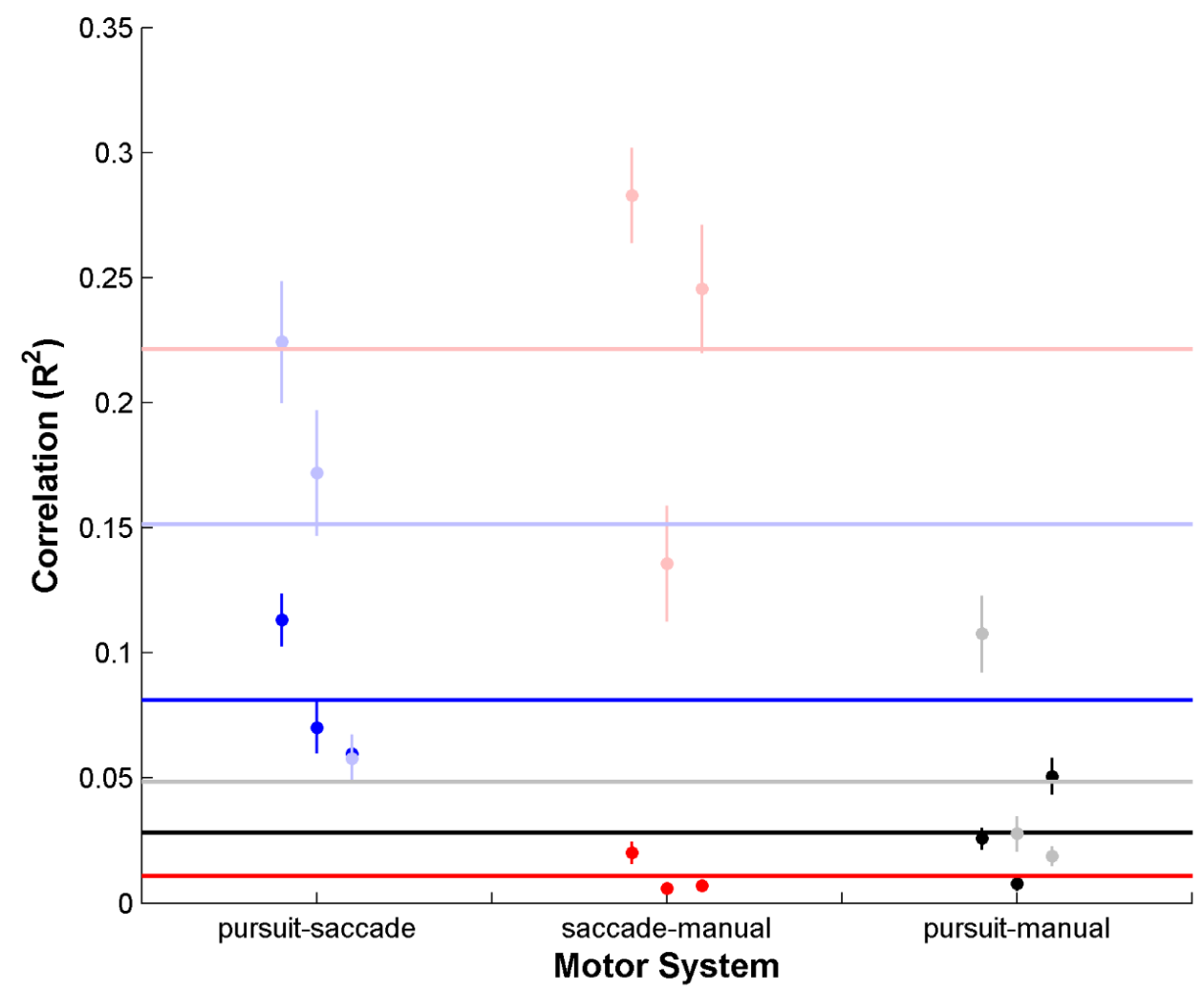

Figure 4. Within-session and across-session shared motor system variability. Horizontal lines represent the average shared variability between pursuit and saccadic (blue), saccadic and manual (red), and pursuit and manual (black) motor systems for all observers across-sessions (unsaturated) and within-session (saturated). Error bars represent the standard deviation of a set of boot-strapped medians per session for across-session (saturated) and across the means of 20 sessions for within-session (solid) squared correlations per observer.

To quantify across-session RT variability, we measured median RT for each session and motor system. This yielded a set of 180 measurements ( 3 observers $\mathrm{x} 3$ motor systems x 20 sessions). Since our RT data did not follow a Gaussian distribution, we calculated across-session RT variability by subtracting the $25^{\text {th }}$ from the $75^{\text {th }}$ percentile and taking the mean. To quantify within-session RT variability, we used the $50^{\text {th }}$ percentile value of the set of medians, collapsing across all 20 sessions for each 
motor system and observer. To quantify shared RT variability between motor systems, we calculated Pearson's $r$ for each pair of motor systems (see Figure 4).

\section{Results and Discussion}

First, we measured the median RT for each of the three motor systems, for each observer. Consistent with well-known values for the timing of smooth pursuit (125-180 ms; (Rashbass, 1961; Robinson, 1964)), saccadic (200-250 ms; (Rashbass, 1961; Robinson, 1965)), and manual button-press responses (350-450 ms; (Welford, 1980)), we observed an average RT of $157 \mathrm{~ms}$ for the pursuit system, $210 \mathrm{~ms}$ for the saccadic system, and $400 \mathrm{~ms}$ for the manual button-press system (see Table 1), collapsing across all values of stimulus contrast.

Table 1

Across and within-session variability in motor RT for Experiment 1

\begin{tabular}{|c|c|c|c|c|c|c|c|c|c|}
\hline \multicolumn{10}{|c|}{ Experiment 1} \\
\hline & \multicolumn{3}{|c|}{ Saccades (ms) } & \multicolumn{3}{|c|}{ Manual (ms) } & \multicolumn{3}{|c|}{ Pursuit (ms) } \\
\hline & median & $\begin{array}{l}\text { across- } \\
\text { session }\end{array}$ & $\begin{array}{l}\text { within- } \\
\text { session }\end{array}$ & median & $\begin{array}{l}\text { across- } \\
\text { session }\end{array}$ & $\begin{array}{l}\text { within- } \\
\text { session }\end{array}$ & median & $\begin{array}{l}\text { across- } \\
\text { session }\end{array}$ & $\begin{array}{l}\text { within- } \\
\text { session }\end{array}$ \\
\hline Observer 1 & 181 & 5 & 17 & 434 & 21 & 59 & 152 & 5 & 17 \\
\hline Observer 2 & 208 & 8 & 24 & 395 & 20 & 49 & 152 & 8 & 24 \\
\hline Observer 3 & 239 & 7 & 24 & 373 & 10 & 62 & 167 & 11 & 25 \\
\hline
\end{tabular}

We then plotted median RT as a function of stimulus contrast for each motor system and observer across sessions, as shown in Figure 2. Using a two-way repeatedmeasures ANOVA, we observed a significant main effect of stimulus contrast $F(5,10)=$ 
$130.50, \mathrm{p}<0.0001$ and motor system $F(2,4)=55.51, \mathrm{p}=0.0012$ on $\mathrm{RT}$, as well as a significant interaction between contrast and motor system $F(5,10)=19.60, \mathrm{p}<0.0001$ on $\mathrm{RT}$, indicating that contrast-RT functions for the three motor systems do not share the same slope. Thus, we fit a two-parameter linear function (Carpenter, 2004; Hick, 1952; Hyman, 1953) to contrast-RT data for each observer, for each motor system, shown as solid lines in Figure 2. Using the multiple independent measurements of across-session variability offered by our six contrast levels, we observed that the across-session RT variability in manual button-press response is larger $(M=17, \mathrm{SD}$ range $=10-20 \mathrm{~ms})$ than either pursuit ( $M=8$, SD range: $5-11 \mathrm{~ms})$ or saccadic responses ( $M=7$, SD range: $5-8$ ms) across sessions $F(2,8)=5.88, \mathrm{p}=0.038$, suggesting that the timing in the manual system is more variable compared to the timing in the pursuit or saccadic system.

Effects of contrast on RT. To quantify the effect of stimulus strength on motor RT we correlated reciprocal RT with log contrast level (Carpenter, 1981). For each motor system, we plotted reciprocal latency for each session as a function of log contrast and quantified Pearson's r (see Figure 3A-C), yielding a set of 180 correlation strengths ( 3 subjects $x 3$ motor systems $x 20$ sessions). For all observers, saccadic response timing was more strongly correlated with stimulus strength (Observer 1: $\mathrm{r}=0.72$; Observer 2: $\mathrm{r}$ $=0.68$; Observer $3: \mathrm{r}=0.55$; all $\mathrm{p}<0.001)$ than either pursuit (Observer $1: \mathrm{r}=0.47$;

Observer 2: $\mathrm{r}=0.37$; Observer 3: $\mathrm{r}=0.16$, all $\mathrm{p}<0.001$ ) or manual button-press timing (Observer 1: $\mathrm{r}=0.19$; Observer 2: $\mathrm{r}=0.18$; Observer 3: $\mathrm{r}=0.15$, all $\mathrm{p}<0.001$ ). Indeed, saccadic RT decreased as stimulus contrast increased. Thus, this horizontally-moving, vertically-offset stimulus provoked saccadic responses with a tighter correlation to 
stimulus contrast than pursuit or manual responses $F(2,8)=15.87, \mathrm{p}=0.004$, suggesting that saccadic RT is more sensitive to changes in stimulus strength.

Shared variability across motor systems. To assess whether shared acrosssession factors (e.g., circadian fluctuation, sleep quality, autonomic arousal, blood sugar, etc.) influence these motor systems in parallel, we quantified the pairwise correlations between median latency measurements for each set of motor systems. This measurement quantifies the strength of factors that change on timescales longer than an individual session. Thus, we observed an average across-session variance of $\left(\mathrm{r}^{2}=0.22\right)$, with saccades and manual button-press responses sharing $22 \%$ of the variance $t(107)=11.17$, $\mathrm{p}<0.0001,\left(\mathrm{r}^{2}=0.15\right)$ with pursuit and saccades sharing $15 \%$ of the variance $t(107)=$ 15.64, $\mathrm{p}<1.0001$, and $\left(\mathrm{r}^{2}=0.05\right)$ with pursuit and manual button-press responses sharing $5 \%$ of the variance $t(107)=7.56, \mathrm{p}<0.0001$. Each pair of motor systems showed significant shared variability across multiple timescales. Thus, the relative strength of across-session correlations to changes in stimulus contrast may be useful to understand how factors that change on a longer timescale (e.g., fatigue, heart rate, circadian rhythm, etc.) impact perceptual and motor performance.

Variability within and across-sessions. To assess trial-by-trial RT variability within an individual session, we fit the reciprocal RT measurements for each motor system with a linear function of log contrast (e.g., Carpenter, 1981; Eq. 1). These residuals quantify RT variability that cannot be accounted for by the stimulus, and which may be due to any number of factors that fluctuate on timescales of one hour or less (e.g., heart rate, blood pressure, fatigue). Thus, we made these measurements to compare the 
relative strength of factors that may influence the timing of motor responses on various timescales (e.g., days or weeks versus minutes or hours). Using Wilcoxon Rank-Sum test, we observed that across-session variance is significantly smaller than within-session variance $W(9,9)=45, p<0.0001$. We conclude that shared variability that influences the timing of motor responses on a trial-by-trial basis has a larger effect than shared variability that influences motor responses across sessions, although both are significant.

Experiment 1 required a left-right, and above-below decision on the part of the observer (e.g., a YES-NO task) with stimulus strength that varied over a large range (5\% to $100 \%$ ), making stimulus contrast the largest factor contributing to RT, especially compared to residual variability (see Figure 2). To facilitate measurement of potential internal noise contributions to RT, we used a two-alternative forced-choice (2-AFC) luminance discrimination task (Experiment 2) with stimulus strength that varied over a much smaller range (0-10 d' units above the background noise).

\section{CHAPTER 3}

\section{EXPERIMENT 2}

Experiment 1 demonstrated that with a high range of stimulus contrast, changes in stimulus strength only accounted for a portion of the RT variability. Thus, we used a smaller range of stimulus luminance values in Experiment 2 to facilitate measurement of potential internal noise contributions. The proposed analysis was performed on data

previously collected to quantify noise in RT (Liston \& Stone, 2013). We used the data to quantify internal noise and shared variance in RT of the saccadic and manual motor 
systems to changes in stimulus luminance. We used a small range of stimulus luminance values (0-10 d' above the background noise) on a 2-AFC RT task. Our stimuli required observers to make a saccade to the brighter of the two stimuli and indicate the chosen stimulus by pressing either the left or right mouse button. Observers were instructed to respond as rapidly and accurately as possible. The hardware used in Experiment 2 was the same as the one used for Experiment 1.

\section{Method}

Observers. Five adult observers provided informed consent (Appendix A) to participate in the experiment (one male, four females, age range: 23-25 years). Observers were required to have normal or corrected-to-normal, 20/20 visual acuity. Each observer completed a total of 20 sessions, each consisting of 400 trials, although we discarded a session from the data analysis due to missing oculomotor in that session. The protocol used was approved by NASA Ames Research Center Human IRB (Appendix D) and San José State University Human IRB (Appendix E). Our methods adhered to the Helsinki Declaration.

Stimulus. The stimuli consisted of two Gaussian-blurred $(\sigma=1$ pixel) disks of diameter $0.6^{\circ}$ framed by a black bounding box (Liston \& Stone, 2008, 2013) and presented $6^{\circ}$ to the left and right of the fixation point. The stimuli were presented on a background of Gaussian pixel noise $\left(37.6 \pm 8.2 \mathrm{~cd} / \mathrm{m}^{2}\right)$. To avoid prediction of stimulus onset (Luce, 1986; Palmer et al., 2005), fixation duration was drawn from a truncated exponential distribution (200-5000 ms, $M=700 \mathrm{~ms})$. We used a 2-AFC task, in which 
observers were required to make a saccade to the chosen stimulus and a button-press response to indicate the chosen stimulus. To ensure a consistent response threshold on the tradeoff between speed and accuracy, we used an auditory reinforcement tone (100$1000 \mathrm{~Hz}$ ) that incorporated RT (i.e., the size of the reward decreased as latency increased) and accuracy (i.e., only correct trials were rewarded) on individual trials (Liston \& Stone, 2008, 2013; Liston \& Krauzlis, 2005). For correct trials, auditory tone and monetary reward were contingent upon RT with the tone and monetary reward decreasing as latency increased.

Procedure. Observers sat in front of the EIZO FlexScan T966 monitor on a bite bar to keep the head relatively stable. On each trial, observers were instructed to fixate a central cross for a randomized duration $(700-5000 \mathrm{~ms})$. After the randomized duration, the fixation disappeared and the stimulus was presented $6^{\circ}$ to the left and right of the fixation point. Observers were instructed to make a saccade to the brighter of the two disks and to make a manual button-press response to indicate the chosen stimulus (Liston $\&$ Stone, 2008). Thus, this stimulus required a saccadic and manual choice on each trial.

Analysis. Since our RT data were non-parametric, we used the median as a measure of central tendency. To quantify the effect of luminance on saccadic and manual button-press latency, we correlated reciprocal RT with stimulus strength for each session. This yielded a set of 20 correlation measurements for each motor system and observer. To quantify shared RT between motor systems, we correlated saccadic and manual RT across sessions and observers. 
To quantify across-session RT variability, we measured median RT for each session and motor system. This yielded a set of 200 measurements ( 5 observers x 2 motor systems x 20 sessions). Since our RT data did not follow a Gaussian distribution, we calculated across-session RT variability by subtracting the 25 th from the 75 th percentile and taking the mean.

To quantify within-session RT variability, we used the 50th percentile value of the set of medians, collapsing across 20 sessions for each motor system and observer. To quantify shared RT variability between motor systems, we calculated Pearson's $r$ for saccadic and manual RT with stimulus strength and for saccadic and manual RT.

\section{Results and Discussion}

We first measured the median RT for saccadic and manual button-press responses, for each observer, and quantified the across-session variability. Consistent with RTs for Experiment 1 and previous RT measurements, we observed a median RT of $240 \mathrm{~ms}$ for saccades and $412 \mathrm{~ms}$ for manual responses (see Table 2). These measurements correspond with $200 \mathrm{~ms}$ for saccades and $400 \mathrm{~ms}$ for manual RTs in Experiment 1. 
Table 2

Across and within-session variability in motor RT for Experiment 2

\begin{tabular}{lcccccc}
\hline Experiment 2 & \multicolumn{5}{c}{ Manual (ms) } \\
\hline \multicolumn{3}{c}{ Saccades (ms) } \\
\hline median & across- & within- & median & across- & within- \\
& & session & session & & session & session \\
\hline Observer 1 & 227 & 7 & 41 & 474 & 9 & 69 \\
Observer 2 & 235 & 3 & 24 & 387 & 11 & 45 \\
Observer 3 & 230 & 6 & 36 & 386 & 10 & 56 \\
Observer 4 & 245 & 5 & 34 & 401 & 13 & 83 \\
Observer 5 & 266 & 16 & 45 & 414 & 24 & 74 \\
\hline
\end{tabular}

Effects of luminance on RT. To quantify the effect of luminance on saccadic and manual button-press latency, we correlated reciprocal RT with stimulus strength (Carpenter, 1981). For each motor system, we plotted reciprocal latency as a function of stimulus strength and quantified Pearson's r (see Figure 5), yielding a set of 200 correlation strengths ( 5 observers x 2 motor systems x 20 sessions) for each observer. Across observers, saccades were better correlated with the stimulus ( $\mathrm{r}=0.18$, paired t-test, $\mathrm{p}<0.0001$ ), decreasing RT as luminance strength increased, compared to manual buttonpress responses $(\mathrm{r}=0.13$, paired t-test, $\mathrm{p}<0.05)$. Thus, our low-luminance stimulus provoked saccadic responses that were better correlated with changes in stimulus strength than manual button-press responses, suggesting that saccadic RT is more sensitive to Just Noticeable Differences (JND) in stimulus strength as compared to manual RT. 

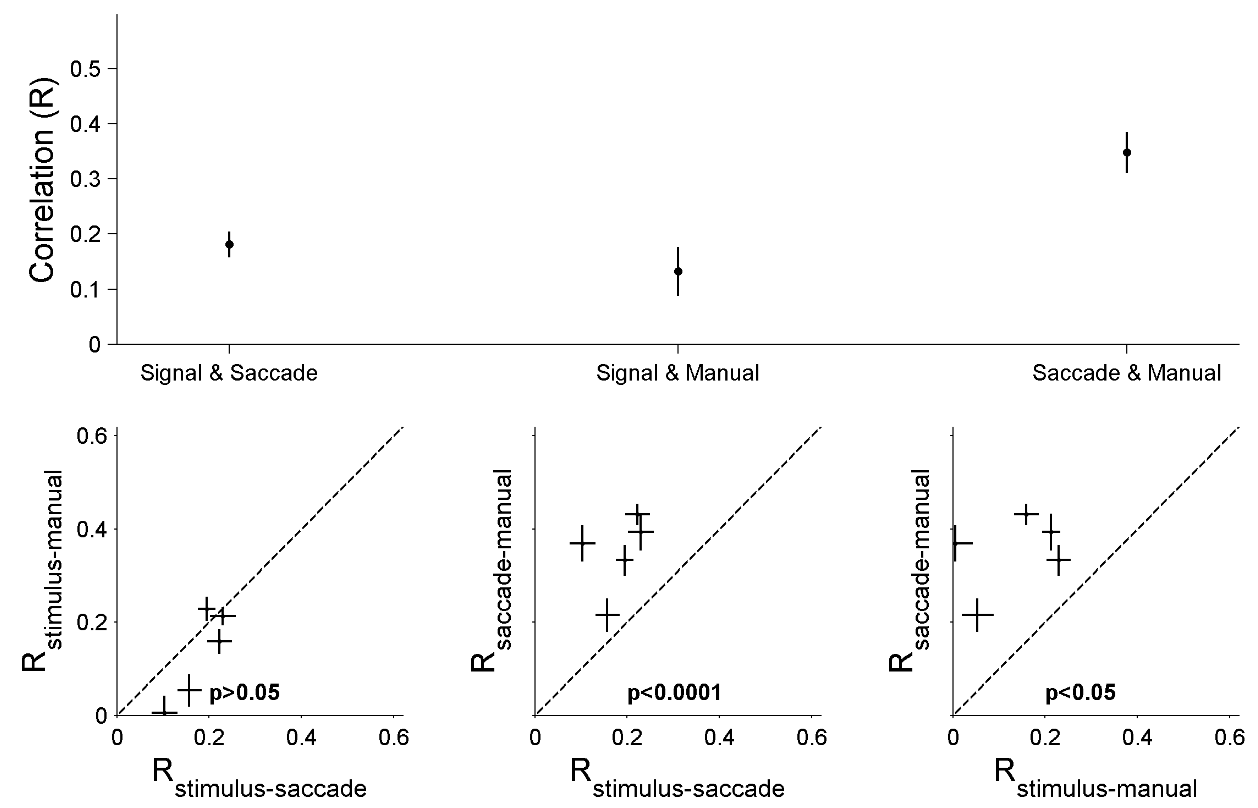

Figure 5. Shared across-session variability between stimulus strength and RT. Filled circles in the top-panel plot shared variability between stimulus strength and saccade response, signal strength and manual response, and saccadic and manual responses. Error bars represent the SEM across observers. Bottom panels compare the correlations between the physical stimulus strength and the motor response time and the response time of both motor systems. Across the signal strength range of these stimuli, saccades and manual response times are better correlated with each other than with stimulus strength.

Shared variability across motor systems. Although the timing of saccadic responses are better correlated with the stimulus, when the JND is closer to not being detected (difference between the two disks is not noticeable), saccadic and manual button-press responses are better correlated with each-other $(r=0.35$, paired t-test $\mathrm{p}>0.05$ ) than either individual system with the stimulus, indicating shared internal noise contributing to RT variability. Thus, the use of a task with a restricted range of stimulus strength facilitated measurement of this shared internal noise, which would not have been salient with only Experiment 1. 
Variability Within and Across-sessions. Consistent with Experiment 1, we observed that the across-session RT variability in manual button-press response $(M=13$ $\mathrm{ms}, \mathrm{SD}=6 \mathrm{~ms})$ is larger than the corresponding variability for saccadic response $(M=7$ $\mathrm{ms}, \mathrm{SD}=5 \mathrm{~ms}) W(5,5)=19, \mathrm{p}=0.095$. However, the difference in manual and saccadic RT is not significant and may be due to the restricted range of stimulus strength. Lastly, the trial-by-trial variability in a session for saccadic and manual responses is greater than variability across all sessions $W(10,10)=55.5, \mathrm{p}<0.0001$, suggesting that changes on a trial-by-trial variability is greater than aggregated variability across all sessions.

\section{CHAPTER 4}

\section{GENERAL DISCUSSION}

Interest in motor RTs arose from the difference in recorded timing of stellar transits in the astronomy community. F. W. Bessel noticed that his timing of when a star crossed the hairline of his telescope was consistently different compared to the timming recorded by a visiting astronomer, with his timing always slightly sooner than the timing of the visiting scholar (Welford, 1980). Intrigued by the difference, he conducted an experiment on the accuracy of the timing and came up with what he called, personal equation, which can be referred to as idiosyncratic differences in the timing of responses between two people. Since then, research in motor RTs has increased and is becoming an objective measurement of changes in neural states (Basner et al., 2011; Dinges \& Powell, 1985; Liston \& Stone, 2014; Pearson et al., 2007). 
Building on RT research pioneered by Helmholtz and Dodge, researchers began to quantify the inverse relationship between response time and changes in stimulus strength (Carpenter, 1981; Cattell, 1886; Piéron, 1927), which for stimuli with a high range in contrast or luminance, RT predictably decreases as stimulus strength increases (Carpenter, 1981; Cattell, 1886; Piéron, 1927) and vice versa. However, interest in the predictability of neural states using RTs fell off and picked up only after the application of information theory to choice RT paradigms (Hicks \& Birren, 1970; Hyman, 1953), which again became a quantifiable measurement of neural states (Carpenter, 1981; Luce, 1986; Welford, 1980).

Traditionally, RT studies used either verbal (Jastrow, 1890; Masson et al., 1994) or manual responses (Basner et al., 2011; Cattell, 1886; Dinges \& Powell, 1985; Piéron, 1927; Wilkinson \& Houghton, 1982). However, as oculomotor measurements became more precise (Yarbus, 1967), interest in oculomotor RTs increased (Carpenter, 1981; Diefendorf \& Dodge, 1908). The advancement of better optical tools allowed for the measurement of other properties of motor behavior that may be useful indicators of changes in neural states (e.g., response time variability, changes in saccadic peak velocity, fixation duration). However, across motor systems, the properties and sensitivity to detect changes in neural states and ultimately serve as a reliable metric for detection and prediction remains a topic of debate. The data in this paper demonstrate that 1) saccadic RTs are better correlated with changes in stimulus strength, 2) manual responses show more variability compared to oculomotor responses, 3) across-session 
variance for all motor systems is smaller than trial-by-trial variability, and 4) shared variability between oculomotor and manual responses indicate shared neural processes.

\section{Effect of Stimulus Strength}

Our data demonstrate that the timing of saccadic responses are better correlated with changes in stimulus strength as compared to pursuit and manual responses. This holds to be true for the stimuli in Experiment 1, which has a large range in stimulus contrast (5-100\%) and for Experiment 2, which has a small range in stimulus luminance (0-10 d' above the background). The correlation between stimulus strength and saccadic RT cannot only be attributed to the general speed of the saccadic system because the pursuit system is indeed faster. However, there is a speed-accuracy tradeoff in the response between the saccadic and pursuit systems (Liston \& Krauzlis, 2003, 2005). Indeed, it has been shown that the pursuit system employs a faster response to the chosen stimulus, but can often be wrong. In cases when the pursuit system is wrong, it makes a corrective turn-around motion and selects the same target as the saccadic response (Liston \& Krauzlis, 2003). Thus, between the two oculomotor systems, though not the fastest to respond, the saccadic system is the most accurate.

The difference in the oculomotor and manual RTs is a bit more elusive. The difference in timing between the motor systems cannot be accounted for only by nerve distance and conduction (Carpenter, 1999; Helmholtz, 1850). Though it seems reasonable that the manual system should take longer to respond to visual stimuli because the nerves travel farther, that is not the case. There is a speed-accuracy tradeoff between 
the saccadic and manual systems that may be driving this difference (Beutter et al., 2003). Correcting a mistake made by poor planning in the manual system can be a lot more costly than a mistake in the oculomotor system. Take, for instance, batting a curve ball, if the batter programmed the manual response before realizing that the ball's trajectory is curved, reprogramming the response is lengthy ( $400 \mathrm{~ms})$ and costly (might not hit the ball). However, in this case, the saccadic system is both faster and better correlated with stimulus strength. Our results suggest that the oculomotor system, in particular the saccadic system, is more sensitive to changes in stimulus strength compared to the pursuit and manual systems.

\section{Motor System Variability}

Variability in the timing across motor systems and within a single system has been an area of interest since Helmholtz measured nerve conduction (Helmholtz, 1850) and subsequent research showed that nerve conduction is only a small portion of the timing in RT (Carpenter, 1981, 1999). Hence, most of the time it takes to respond to a stimulus is occupied by central processes (Carpenter, 1999; Welford, 1980). In some cases, the response within a motor system is more variable. If one is to use the RT of one motor system to measure internal changes, how much variability should that system have?

Our data show that variability in manual responses is larger than variability in either of the two oculomotor systems. Since the sensory input for oculomotor and manual systems is the same and nerve conduction is not the reason why manual responses 
take longer and are more variable, a difference in the decision-making mechanism might be responsible for the observed difference. Most of the time taken by the saccadic system to respond to a stimulus is occupied up by higher-level structures (Carpenter, 1999) involved in processes determining what is most relevant and important. The manual system, having to program a more complex response, receives input and inhibition from more structures. Thus, there are two ways of thinking about the variability in the timing of the manual motor system. One is that the difference could be due to a greater sensitivity to factors that fluctuate across sessions (e.g., fatigue, circadian factors, sleep deprivation, sympathetic system arousal). The second is simply more variability in the timing of the motor response due to the number of structures involved in programming and executing the command, which suggests greater noise in general.

Consider using the responses of one of the motor system to detect changes in fatigue (Jastrow, 1888; Ting et al., 2008; Welford, 1980), sleep deprivation (Basner et al., 2011; Dinges \& Powell, 1985; Heaton et al., 2014; Lisper \& Kjellberg, 1972), or brain injuries (Heitger et al., 2004). On the one hand, the relatively high within and acrosssession variability of manual button-press responses may impede detection of small RT changes related to neural states or brain injury. On the other hand, the relatively large across-session variability may be related to meaningful performance fluctuations (e.g., fatigue) that may have changed across sessions, but will obscure changes in RT related to traumatic brain injuries, for example. Considering the two scenarios and taking into account that both oculomotor systems are better correlated with changes in the stimulus, the manual system's variability seems to be due to fluctuating noise not to meaningful 
changes. In this case, a system with greater inhibition and command from higher-level structures seem to obscure the internal changes we are interested in measuring.

\section{Variability Across Multiple Timescales}

It has been reported that under some conditions, RT and RT variability increase with the severity of the condition (Kobrick \& Dusek, 1970; Kobrick, 1972). Thus, RT variability across multiple timescales (e.g., hour, day, weeks) can give insight to the severity, condition, or even progression of neural states. However, it is not clear whether the difference can be measured in an individual session (hour) or across-sessions (months). For our data, responses across all motor systems show greater variability within the course of a session than across all sessions. For all motor systems, the median RT for a particular observer tends to be very stable compared to trial-by-trial variability. This observation poses the question, why is variability within a session greater than variability across sessions?

Previous discussions of within-session variability have noted this "gratuitous randomization" inherent in RT as potentially conferring evolutionary advantages (Carpenter, 1981, 1999). For detection of changes in performance, however, the median RT provides a stable summary metric, although the within-session RT variability may also be useful, especially in the context of Carpenter's LATER model, in which a decision signal rises as a response to incoming sensory evidence until a threshold is achieved and a response initiated (Carpenter, 1999). However, the rate of rise in response to sensory information fluctuates from trial-to-trial in a manner that cannot be 
accounted for by nerve conduction or synaptic delay (Carpenter, 1999), suggesting that the "gratuitous randomization" serves as an internal process that randomizes the response. Thus, a single session may be enough to give insight to the immediate internal state while multiple sessions may show stability/ instability, or progression.

\section{Shared Neural Noise}

Lastly, RT correlations between all pairs of motor systems show shared internal noise. This finding is not surprising for the oculomotor systems (Liston \& Krauzlis, 2003; Liston \& Stone, 2013). However, the shared internal noise between manual button-press and saccadic responses was only apparent with the stimulus with a low range in luminance values, which was likely measuring internal variability impacting the timing of both motor systems in conjunction. Indeed, shared neural noise between oculomotor and manual responses, despite their differences in response timing, have only recently been documented (Beutter et al., 2003; Liston \& Krauzlis, 2003, 2005; Stone \& Krauzlis, 2003).

Thus, a stimulus with a large range in strength such as contrast in Experiment 1, is likely measuring variability in the sensory input stage. However, a stimulus with low detectability such as the stimulus in Experiment 2, is likely measuring variability in the response preparation or the decision stage. Our data demonstrates that under conditions where stimulus strength is low, saccadic and manual button-press responses are better correlated with each other than either one with the stimulus (Beutter et al., 2003), suggesting a shared process that influences the response preparation or decision stage. 
Furthermore, we observed significant positive correlations among all three motor systems across sessions for one observer indicating that under some behavioral conditions, shared across-session variability contributes to the timing of all three motor systems.

In summary, the saccadic system is more tightly correlated with changes in stimulus strength as compared to the pursuit and manual systems, suggesting that it is more sensitive to stimulus properties. In addition, our stimulus produced manual responses with greater variability than the either oculomotor systems, suggesting that the manual system is noisier and more variable. Also, trial-by-trial variability is greater than variability across sessions for all motor systems, indicating that all three motor systems show "gratuitous randomization" in responding to a stimulus. Lastly, shared variability within pairs of motor systems suggests shared neural processes and noise. Taking into consideration the data presented in this thesis, the motor system best suited for the detection of changes to internal states is the saccadic system. The saccadic system is better correlated with changes in stimulus strength, more predictable, and less variable as compared to the pursuit and manual RT systems. 


\section{References}

Abadi, R. V., \& Gowen, E. (2004). Characteristics of saccadic intrusions. Vision Research, 44, 2675-2690. http://doi.org/10.1016/j.visres.2004.05.009

Anderson, T. J., \& MacAskill, M. R. (2013). Eye movements in patients with neurodegenerative disorders. Nature Reviews. Neurology, 9, 74-85. http://doi.org/10.1038/nrneurol.2012.273

Bartlett, N. R., \& Macleod, S. (1954). Effect of flash and field luminance upon human reaction time. Journal of the Optical Society of America, 44, 306-311. http://doi.org/10.1364/JOSA.44.000306

Basner, M., Mollicone, D., \& Dinges, D. F. (2011). Validity and sensitivity of a brief psychomotor vigilance test (PVT-B) to total and partial sleep deprivation. Acta Astronautica, 69, 949-959. http://doi.org/10.1016/j.actaastro.2011.07.015

Beutter, B. R., Eckstein, M. P., \& Stone, L. S. (2003). Saccadic and perceptual performance in visual search tasks. I. Contrast detection and discrimination. Journal of the Optical Society of America. A, Optics, Image Science, and Vision, 20, 13411355. http://doi.org/10.1364/JOSAA.20.001341

Beutter, B. R., \& Stone, L. S. (1998). Human motion perception and smooth eye movements show similar directional biases for elongated apertures. Vision Research, 38, 1273-1286. http://doi.org/10.1016/S0042-6989(97)00276-9

Beutter, B. R., \& Stone, L. S. (2000). Motion coherence affects human perception and pursuit similarly. Visual Neuroscience, 17, 139-153. http://doi.org/10.1017/S0952523800171147

Bompas, A., \& Sumner, P. (2008). Sensory sluggishness dissociates saccadic, manual, and perceptual responses: an S-cone study. Journal of Vision, 8, 10.1-13. http://doi.org/10.1167/8.8.10

Buck, L. (1966). Reaction time as a measure of perceptual vigilance. Psychological Bulletin, 65, 291-304. http://doi.org/10.1037/h0023207

Carpenter, R. H. S. (1981). Ocular procrastination. In D. F. Fisher, R. A. Monty, \& J. W. Senders (Eds.), Eye Movements: Cognition and Visual Perception (pp. 237-246). Hillsdale, NJ: Lawrence Erlbaum.

Carpenter, R. H. S. (1999). A Neural Mechanism that Randomises Behavior. Journal of 
Consciousness Studies, 6, 13-22.

Carpenter, R. H. S. (2004). Contrast, pobability, and saccadic latency: Evidence for independence of detection and decision. Current Biology, 14, 1576-1580. http://doi.org/10.1016/j.cub.2004.08.058

Cattell, J. M. (1886). Thw influence of the intensity of the stimulus on the length of the reaction time. Brain, 8, 512-515.

Diefendorf, A. R., \& Dodge, R. (1908). An experimental study of the ocular reactions of the insane from photographic records. Brain, 31, 451-489. http://doi.org/10.1093/brain/31.3.451

Dinges, D. F., \& Powell, J. W. (1985). Microcomputer analyses of performance on a portable, simple visual RT task during sustained operations. Behavior Research Methods, Instruments, and Computers, 17, 652-655.

Duschek, S., Weisz, N., \& Schandry, R. (2003). Reduced cognitive performance and prolonged reaction time accompany moderate hypotension. Clinical Autonomic Research : Official Journal of the Clinical Autonomic Research Society, 13, 427432. http://doi.org/10.1007/s10286-003-0124-4

Flykt, A. (2005). Visual search with biological threat stimuli: accuracy, reaction times, and heart rate changes. Emotion (Washington, D.C.), 5, 349-353. http://doi.org/10.1037/1528-3542.5.3.349

Fowler, B., \& Prlic, H. (1995). A comparision of visul and auditory reaction time and P300 latency thresholds to acute hypoxia. Aviation, Space, and Environmental Medicine, 66, 645-650.

Garbutt, S., Matlin, A., Hellmuth, J., Schenk, A. K., Johnson, J. K., Rosen, H., ... Boxer, A. L. (2008). Oculomotor function in frontotemporal lobar degeneration, related disorders and Alzheimer's disease. Brain, 131, 1268-1281. http://doi.org/10.1093/brain/awn047

Green, D. A., \& Swets, J. A. (1966). Signal detection theory and psychophysics. John Wiley \& Sons, Inc.

Heaton, K. J., Maule, A. L., Maruta, J., Kryskow, E. M., \& Ghajar, J. (2014). Attention and visual tracking degradation during acute sleep deprivation in a military sample. Aviation Space and Environmental Medicine, 85, 497-503.

http://doi.org/10.3357/ASEM.3882.2014 
Heitger, M. H., Anderson, T. J., Jones, R. D., Dalrymple-Alford, J. C., Frampton, C. M., $\&$ Ardagh, M. W. (2004). Eye movement and visuomotor arm movement deficits following mild closed head injury. Brain, 127, 575-590. http://doi.org/10.1093/brain/awh066

Helmholtz, H. L. F. (1850). Messungen über den zeitlichen verlauf der zuckung animalischer muskeln und die fortpflanzungsgeschwindigkeit der reizung in den nerven. Archiv Für Anatomie, Physiologie Und Wissenschaftliche Medicin, 276364.

Henmon, V. A. C. (1906). The time of perception as a measure of differences in sensations. New York: Science press.

Hick, W. E. (1952). On the rate of gain of information. Quarterly Journal of Experimental Psychology. http://doi.org/10.1080/17470215208416600

Hicks, L. H., \& Birren, J. E. (1970). Aging, brain damage, and psychomotor slowing. Psychological Bulletin, 74, 377-396.

Hikosaka, O., Nakamura, K., \& Nakahara, H. (2006). Basal ganglia orient eyes to reward. Journal of Neurophysiology, 95, 567-584. http://doi.org/10.1152/jn.00458.2005

Hyman, R. (1953). Stimulus information as a determinant of reaction time. Journal of Experimental Psychology, 45, 188-196. http://doi.org/10.1037/h0056940

Jastrow, J. (1888). A critique of psycho-physic Methods. The American Journal of Psychology, 1, 271-309. http://doi.org/10.1016/0378-8733(91)90003-C

Jastrow, J. (1890). The time-relations of mental phenomena. Science (New York, N.Y.), 16, 155-157. http://doi.org/10.1126/science.ns-16.398.155

Jennings, J. R., \& Wood, C. C. (1977). Cardiac cycle time effects on performance, phasic cardiac responses, and their intercorrelation in choice reaction time. Psychophysiology, 14, 297-307.

Kane, A., Wade, A., \& Ma-Wyatt, A. (2011). Delays in using chromatic and luminance information to correct rapid reaches. Journal of Vision, 11, 1-18. http://doi.org/10.1167/11.10.3

Kobrick, J. L. (1972). Effects of hypoxia on voluntary response time to peripheral stimuli during central target monitoring. Ergonomics, 15, 147-156. http://doi.org/10.1080/00140137208924419

Kobrick, J. L., \& Dusek, E. R. (1970). Effects of hypoxia on voluntary response time to 
peripherally located visual stimuli. Journal of Applied Physiology (Bethesda, Md. : 1985), 29, 444-448.

Krauzlis, R. J., \& Adler, S. a. (2001). Effects of directional expectations on motion perception and pursuit eye movements. Visual Neuroscience, 18, 365-376. http://doi.org/10.1017/S0952523801183033

Krauzlis, R. J., \& Stone, L. S. (1999). Tracking with the mind's eye. Trends in Neurosciences, 22, 544-550. http://doi.org/10.1016/S0166-2236(99)01464-2

Liguori, A., Gatto, C. P., \& Robinson, J. H. (1998). Effects of marijuana on equilibrium, psychomotor performance, and simulated driving. Behavioural Pharmacology, 9, 599-609. http://doi.org/10.1097/00008877-199811000-00015

Lisper, H. O., \& Kjellberg, a. (1972). Effects of 24-hour sleep deprivation on rate of decrement in a 10-minute auditory reaction time task. Journal of Experimental Psychology, 96, 287-290. http://doi.org/10.1037/h0033615

Liston, D. B., \& Krauzlis, R. J. (2003). Shared response preparation for pursuit and saccadic eye movements. The Journal of Neuroscience: The Official Journal of the Society for Neuroscience, 23, 11305-11314. http://doi.org/23/36/11305 [pii]

Liston, D. B., \& Krauzlis, R. J. (2005). Shared decision signal explains performance and timing of pursuit and saccadic eye movements. Journal of Vision, 5, 678-689. http://doi.org/10.1167/5.9.3

Liston, D. B., Krukowski, A. E., \& Stone, L. S. (2013). Saccade detection during smooth tracking. Displays, 34, 171-176. http://doi.org/10.1016/j.displa.2012.10.002

Liston, D. B., \& Stone, L. S. (2008). Effects of prior information and reward on oculomotor and perceptual choices. The Journal of Neuroscience: The Official Journal of the Society for Neuroscience, 28, 13866-13875. http://doi.org/10.1523/JNEUROSCI.3120-08.2008

Liston, D. B., \& Stone, L. S. (2013). Saccadic brightness decisions do not use a difference model. Journal of Vision, 13, 1-10. http://doi.org/10.1167/13.8.1.doi

Liston, D. B., \& Stone, L. S. (2014). Oculometric assessment of dynamic visual processing. Journal of Vision, 14, 1-17. http://doi.org/10.1167/14.14.12.doi

Luce, D. R. (1986). Response Times. (D. E. Broadbent, J. L. McGaugh, N. J. Mackintosh, M. I. Posner, E. Tulving, \& L. Weiskrantz, Eds.). New York, New York: Oxford University Press. 
Martin, E. M., Robertson, L. C., Edelstein, H. E., Jagust, W. J., Sorensen, D. J., San Giovanni, D., \& Chirurgi, V. A. (1992). Preformance of patients with early HIV-1 infection on the stroop task. Journal of Clinical and Experimental Neuropsychology, $14,857-868$.

Masson, G., Mestre, D. R., Blin, O., \& Pailhous, J. (1994). Low luminance contrast sensitivity: Effects of training on psychophysical and optokinetic nystagmus thresholds in man. Vision Research, 34, 1893-1899. http://doi.org/10.1016/00426989(94)90313-1

Miller, J. C. (2004). The sensitivity and specificity of oculometric under fatigue stress, compared to performance and subjective measures. Brooks-City-base.

Palmer, J., Huk, A. C., \& Shadlen, M. N. (2005). The effect of stimulus strength on the speed and accuracy of a perceptual decision. Journal of Vision, 5, 376-404. http://doi.org/10.1167/5.5.1

Pearson, B. C., Armitage, K. R., Horner, C. W. M., \& Carpenter, R. H. S. (2007). Saccadometry: the possible application of latency distribution measurement for monitoring concussion. British Journal of Sports Medicine, 41, 610-612. http://doi.org/10.1136/bjsm.2007.036731

Piéron, H. (1927). L’influence de l'intensité sur le temps de réaction a' la cessation du stimulus luminem. Compte Rendu de La Societé de Biologie, 97, 1147-1149.

Rashbass, C. (1961). The relationship between saccadic and smooth tracking eye movements. The Journal of Physiology, 159, 326-338. http://doi.org/10.1016/01969781(92)90172-Y

Ratino, D. A., Repperger, D. W., Goodyear, C., Potor, G., \& Rodriguez, L. E. (1988). Quantification of reaction time and time perception during Space Shuttle operations. Aviation, Space, and Environmental Medicine, 59, 200-204.

Robinson, D. A. (1964). The mechanics of human saccadic eye movement. The Journal of Physiology, 174, 245-264.

Robinson, D. A. (1965). The mechanics of human smooth pursuit eye movement. The Journal of Physiology, 180, 569-591. http://doi.org/VL - 180

Rogers, N. L., Phan, O., Kennaway, D. J., \& Dawson, D. (1998). Effect of daytime oral melatonin administration on neurobehavioral performance in humans. Journal of Pineal Research, 25, 47-53. http://doi.org/10.1111/j.1600-079X.1998.tb00385.x 
Sahin, L., Wood, B. M., Plitnick, B., \& Figueiro, M. G. (2014). Daytime light exposure: Effects on biomarkers, measures of alertness, and performance. Behavioural Brain Research, 274, 176-185. http://doi.org/10.1016/j.bbr.2014.08.017

Stone, L. S., \& Krauzlis, R. J. (2003). Shared motion signals for human perceptual decisions and oculomotor actions. Journal of Vision, 3, 725-736. http://doi.org/10.1167/3.11.7

Taylor, M. J., Carpenter, R. H. S., \& Anderson, a J. (2006). A noisy transform predicts saccadic and manual reaction times to changes in contrast. The Journal of Physiology, 573, 741-751. http://doi.org/10.1113/jphysiol.2006.105387

Ting, P. H., Hwang, J. R., Doong, J. L., \& Jeng, M. C. (2008). Driver fatigue and highway driving: A simulator study. Physiology and Behavior, 94, 448-453. http://doi.org/10.1016/j.physbeh.2008.02.015

Tzambazis, K., \& Stough, C. (2000). Alcohol impairs speed of information processing and simple and choice reaction time and differentially impairs higher-order cognitive abilities. Alcohol and Alcoholism (Oxford, Oxfordshire), 35, 197-201. http://doi.org/10.1016/S0924-977X(99)80540-9

Uri, J. J., Linder, B. J., Moore, T. P., Pool, S. L., \& Thornton, W. E. (1989). Saccadic Eye Movements During Space Flight. Houston.

Van Stockum, S., Macaskill, M. R., Myall, D., \& Anderson, T. J. (2013). A perceptual discrimination task results in greater facilitation of voluntary saccades in Parkinson's disease patients. European Journal of Neuroscience, 37, 163-172. http://doi.org/10.1111/ejn.12033

Welford, A. T. (1980). Reaction Times. England United Kingdom: Academic Press.

Wheeless, L. L., Cohen, G. H., \& Boynton, R. M. (1967). Luminance as a parameter of the eye-movement control system. Journal of the Optical Society of America, 57, 394. http://doi.org/10.1364/JOSA.57.000394

Wilkinson, R. T., \& Houghton, D. (1982). Field test of arousal: A portable reaction timer with data storage. Human Factors, 24, 487-493. http://doi.org/10.1177/001872088202400409

Yarbus, A. L. (1967). Eye movements and vision (1st ed.). Springer science. http://doi.org/10.1007/978-1-4899-5379-7 


\section{Appendix A}

\section{NASA Ames Minimal Risk Consent Form}

\section{Ames Research Center}

\section{CATEGORY II - HUMAN RESEARCH}

MINIMAL RISK

To the Research Participant: Please read this consent form and the attached protocol and/or subject instructions carefully. Make sure all your questions have been answered to your satisfaction before signing. A. I agree to participate in the research experimentas described in the attached
protocol or subjectinstructions. I understand that I am employed by_ $\quad$ who can be contacted at

B. I understand that my participation could cause me minimal risk*, inconvenience, or discomfort. The purpose and procedures have been explained to me and I understand the risks and discomforts as described in the attached research protocol.

C. To my knowledge, I have no medical conditions, including pregnancy, that will prevent my participation in this study. I understand that if my medical status should change while I am a participant in the research experiment there may be unforeseeable risks to me (or the embryo or fetus if applicable). I agree to notify the Principal Investigator (PI) or medical monitor of any known changes in my condition for safety purposes.

D. My consent to participate has been freely given. I may withdraw my consent, and thereby withdraw from the study at any time without penalty or loss of benefits to which I am entitled.I understand that the PI may request my withdrawal or the study may be terminated for any reason. I agree to follow procedures for orderly and safe termination.

E. I am not releasing NASA or any other organization or person from liability for any injury arising as a result of my participationinthisstudy. I will be contacted by the PI if an unusual or abnormal (anomalous) finding is detected during this study.

F. In the event of injury or illness resulting from this study and calling for immediate action or attention, NASA will provide, or cause to be provided, the necessary emergency treatment. If I am eligible for and receive workers' compensation benefits while participating in this study, I cannot sue my employer because the law makes workers' compensation my only remedy against my employer. I may have other remedies against other persons or organizations, depending on the circumstances of the injury. The United States Government will pay for any claims of injury or loss of life to the extent required by the Federal Employees

Compensation Act or the Federal Tort Claims Act.

G. I hereby agree that all records collected by NASA in the course of this study are available to the research study investigators, support staff, and any duly authorized research review committee. I grant NASA permission to reproduce and publish all records, notes, or data collected from my participation, provided there will be no association of my name with the collected data and that confidentiality is maintained, unless specifically waived by me. All stated precautions will be taken to protect anonymity, but there is a small risk that some or all of the participants'data could become identifiable.

H. I have had an opportunity to ask questions and have received satisfactory answers to all my questions. I understand that the $\mathrm{Pl}$ for the study is the person responsible for this activity and that any questions regarding the research will be addressed to $\mathrm{him} /$ her during the course of the study. I have read the above agreement, the attached protocol and/or subject instructions prior to signing this form and I understand the contents.

- Minimal Risk means that the probability and magnitude of harm or discomfort anticipated in the research are not greater, in and of themselves, than those ordinarily encountered in daily life or during the performance of routine physical or psychological examinations or tests.

Signature of Research Participant Date Signature of Principal Investigator Date

Printed/Typed Name of Research $\quad$ Printed/Typed Name of Principal Investigator

Participant

Telephone Number of Research

Telephone Number of Principal Investigator

Participant

Address

Subject Signature: Authorization for Videotaping

City, Sate, Zip Code 


\section{Appendix B}

\section{NASA Ames Research Center IRB Approval Experiment 1}

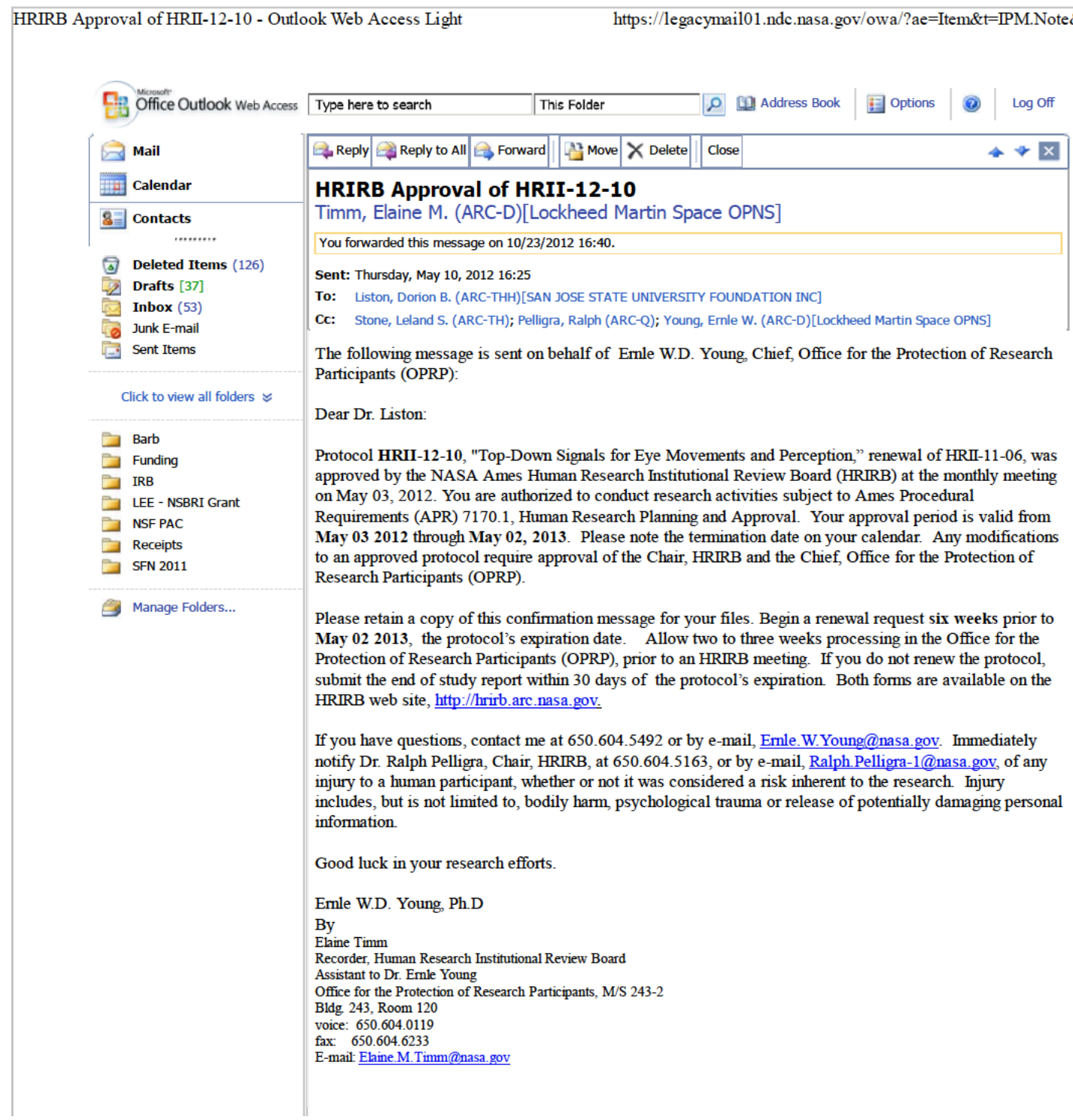




\section{Appendix C}

\section{San Jose Staté University IRB Approval Experiment 1}

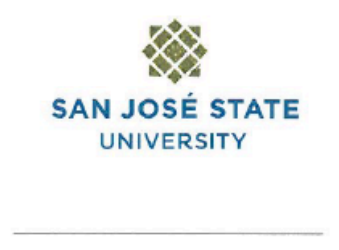

Division of Academic Affairs

Associate Vice President

Graduate Studies \& Research

www.sjsu.edu/gradstudies

One Washington Square

San José, California 95192-0025

Voice: 408-924-2427

Fax 408-924-2612

www.sjsu.edu

\author{
To: Dr. Dorion Liston \\ NASA Ames Research Center \\ Mail Stop 262-2 \\ Moffett Field, CA 94035
}

From: Alena Filip, Institutional Review Board Coordinator Office of Graduate Studies and Research

San Jose State University

One Washington Square

San Jose, CA 95192-0025

Date: $1 / 22 / 14$

Re: Physiological correlates of motor response time

This letter is to inform you that our office has received documentation of your involvement in human subjects research at an outside institution. Your activities have been registered with the San Jose State University IRB and assigned an IRB tracking number: F1402003. Our office has received confirmation of IRB approval for this research from NASA Ames. Because an appropriate IRB approval has already been obtained, no further documents are required at this time. You may proceed with your involvement in the study in accordance with the protocol that was approved by the NASA IRB.

This registration is valid for the duration of the NASA IRB approval.

If you have any questions please contact me at Alena.Filip@sjsu.edu or (408) 924-2479.

Protocol \# F1402003 


\section{Appendix D}

\section{NASA Ames Research Center IRB Approval Experiment 2}

National Aeronautics and Space Administration

Ames Research Center Moffett Field, CA 94035-1000

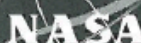

January 14, 2014

Reply to Attn of:

$$
\mathrm{QH} / 243-2
$$

Dorion Liston, Ph.D., Principal Investigator, San Jose State University Foundation; Code TH

FROM: $\quad$ Ralph Pelligra, Chair, NASA Ames Research Center (ARC) Human Research Institutional Review Board (HRIRB)

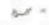

SUBJECT: HRIRB Approval of Protocol HRII-13-12, "Physiological Correlates of Motor Response Time"

Approval Period: May 03, 2013 through May 02, 2014

Protocol HRII-13-12 received approval by expedited review from the Chair, Human Research Institutional Review Board (HRIRB) on May 03, 2013. The approval was ratified by the HRIRB on September 05, 2013.

The Principal Investigator is authorized to conduct research, subject to the requirements as outlined in APR 7170.1, Human Research Planning and Approval. Any modifications to an approved protocol require the further approval of the Chair, HRIRB. Immediately notify the Chair, HRIRB, of any injury to a human participant, whether or not it was considered a risk inherent to the research. Injury includes, but is not limited to, bodily harm, psychological trauma or release of potentially damaging personal information.

Submit a renewal request to the Office for the Protection of Research Participants (OPRP), M/S 243-2, six weeks prior to May 02, 2014, the protocol's expiration date. If you do not renew the protocol, submit an end-of-study report within $\mathbf{3 0}$ days of the protocol's expiration. The forms are available on the HRIRB website, http://hrirb.arc.nasa.gov.

If you have any questions, please contact me at (650)-604-5163 or by e-mail at Ralph.Pelligra-1@nasa.gov. Good luck in your research endeavors.

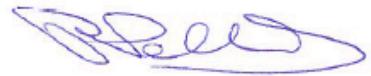

Ralph Pelligra, M.D. 


\section{Appendix E}

\section{San José State University IRB Approval Experiment 2}

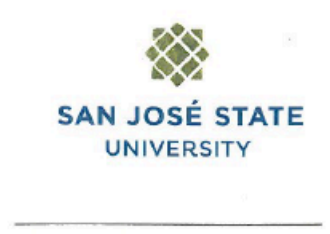

Division of Academic Affairs

Associate Vice President Craduate Studies \& Research

www.sjsu.edu/gradstudies

One Washington Square San Jose, California 95192-0025 Voice: $408-924-2427$

www.sjsu.edu

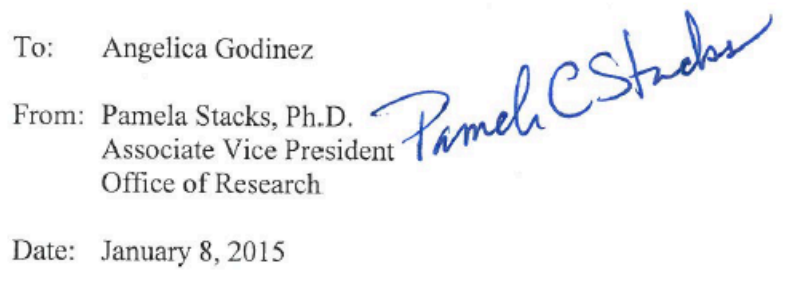

The Human Subjects-Institutional Review Board has registered your study entitled:

"Quantifying variability in oculomotor and manual choice response times"

This registration, which provides exempt status under Exemption Category 4 of SJSU Policy S08-7, is contingent upon the subjects included in your research project being appropriately protected from risk. Specifically, protection of the anonymity of the subjects' identity with regard to all data that may be collected about the subjects from your secondary sources needs to be ensured.

This registration includes continued monitoring of your research by the Board to assure that the subjects are being adequately and properly protected from such risks. If at any time a subject becomes injured or complains of injury, you must notify Dr. Pamela Stacks, Ph.D. immediately. Injury includes but is not limited to bodily harm, psychological trauma, and release of potentially damaging personal information. This approval for the human subject's portion of your project is in effect for one year, and data collection beyond January 8, 2016 requires an extension request.

If you have any questions, please contact me at (408) 924-2427.

Protocol \#: S1404092

cc. Kevin Jordan

0120 